\title{
Sex-determining region Y-box protein 3 induces epithelial-mesenchymal transition in osteosarcoma cells via transcriptional activation of Snail1
}

\author{
Manle Qiu ${ }^{1+}$, Daoyun Chen ${ }^{1 \dagger}$, Chaoyong Shen ${ }^{2 \dagger}$, Ji Shen ${ }^{1}$, Huakun Zhao ${ }^{1}$ and Yaohua He ${ }^{1 *}$
}

\begin{abstract}
Background: The transcription factor sex-determining region Y-box protein 3 (SOX3) plays important roles in various types of cancer. However, its expression and function have not yet been elucidated in osteosarcoma (OS).

Methods: The expression levels of SOX3 in OS tissues and OS cell lines were determined by quantitative real-time polymerase chain reaction (qRT-PCR) and Western blot analysis. The effects of SOX3 expression on OS cell biological traits were investigated by overexpressing and downregulating SOX3 protein. The expression of epithelial-mesenchymal transition (EMT) markers and transcription factors associated with EMT (EMT-TFs), were detected simultaneously. The mechanism underlying SOX3-mediated Snail1 expression was further investigated.

Results: SOX3 was upregulated in human OS tissues. SOX3 overexpression promoted the EMT, migration and invasion in OS cells. The downregulation of SOX3 resulted in opposing effects. Furthermore, SOX3 upregulation enhanced the expression of the transcriptional repressor Snail1 by binding to its promoter region. Additionally, a positive correlation among the expression of SOX3, Snail1, and E-cadherin was demonstrated in human OS tissues.

Conclusions: SOX3 promotes migration, invasiveness, and EMT in OS cells via transcriptional activation of Snail 1 expression, suggesting that SOX3 is a novel regulator of EMT in OS and may serve as a therapeutic target for the treatment of OS metastasis.
\end{abstract}

Keywords: Sex-determining region Y-box protein 3, Snail1, Transcriptional activation, Epithelial-mesenchymal transition, Osteosarcoma

\section{Background}

Osteosarcoma (OS) is one of the most prevalent malignant bone tumors in childhood and adolescence. Similar to most other malignant tumors, OS is characterized by a high propensity for metastasis, which is the leading cause of death [1]. Despite several advancements in neoadjuvant chemotherapies and surgical methods in the treatment of OS, the 5-year survival of patients with metastatic disease is dismal $(<20 \%)$ [2-4]. Therefore, it is necessary to urgently identify new targets or factors

\footnotetext{
* Correspondence: heyaohuadoctor@163.com

${ }^{\dagger}$ Equal contributors

${ }^{1}$ Department of Sports Medicine, Shanghai Jiao Tong University Affiliated

Sixth People's Hospital, 600 Yishan Road, Shanghai 200233, China

Full list of author information is available at the end of the article
}

that govern metastasis and to develop novel therapeutic strategies for OS management.

Epithelial to mesenchymal transition (EMT), a developmental process in which epithelial cells lose polarity and develop a mesenchymal phenotype, plays an important role in the initiation of metastasis [5]. It is believed that EMT endows cancer cells with migratory and invasive properties, and induces cancer stem cell (CSC) properties [6]. The complex genetic changes during EMT are, at least in part, mediated by a number of specific transcription factors, such as Snail, Slug, Twist1, ZEB1, and ZEB2 [7-9]. Snail is overexpressed in various human solid tumors, including numerous types of carcinomas, as well as sarcomas, gliomas, neuroblastomas, and melanomas [10, 11]. Further, increased Snail1 
expression in cancer cells promotes metastatic ability in vivo, as well as cell survival, angiogenesis, and chemoresistance in vitro [12-14]. In addition, exogenous overexpression of Snail1 increases the invasive and metastatic abilities of cancer cells through promoting the downregulation of E-cadherin and EMT [15]. However, the molecular mechanism underlying the upregulation of Snail1 in cancer cells remains unclear.

Sex-determining region Y-box protein 3 (SOX3) is a member of the Sox family of transcription factors that plays an important role in developmental processes, including neurogenesis [16], testis development [17], and chondrogenesis [18], among others [19, 20]. The expression of SOX3 decreases as development proceeds, and correlates with the cellular switch from proliferation to differentiation [21]. Recent studies have reported that SOX3 is upregulated in cancer tissues and may therefore play an oncogenic role in esophageal squamous cell carcinoma, ovarian cancer, and T-cell lymphomas [22-24]. Aberrant SOX3 expression also induces oncogenic transformation of chicken embryonic fibroblasts [25]. However, the function of SOX3 in OS progression has not yet been investigated.

In the present study, we first demonstrate that SOX3 expression is upregulated in human OS tissues and promotes metastatic potential both in vitro and in vivo. SOX3 also induces EMT via enhancing Snail1 expression in OS cells. Moreover, we reveal a positive correlation between SOX3 and Snail1 expression in OS samples.

\section{Methods}

\section{Tissue specimens}

mRNA and protein samples of human OS tissues $(n=42)$, the matched adjacent non-tumor samples $(n=42)$ and bone cysts $(n=28)$ were collected from patients who were admitted to Shanghai Jiao Tong University Affiliated Sixth People's Hospital between 2009 and 2014. The adjacent non-tumor sample were resected within at least $5 \mathrm{~cm}$ of the tumor margin when the patients underwent definitive surgery. Pathological white slides of OS tissues and clinical information were obtained from 18 patients who were admitted to the cohort. Ethical approval for study was provided by the ethics committee of Shanghai Jiao Tong University Affiliated Sixth People's Hospital. Written informed consent was obtained from all subjects or their guardians.

\section{Cell lines and cell culture}

U2OS, SoSP-M, SoSP-9607, and MG-63 cells were purchased from the Cell Bank of China Academy of Sciences (Shanghai, China) and routinely checked for Mycoplasma contamination by Hoechst staining. U2OS, SoSP-M, SoSP-9607 were cultured in RPMI 1640 medium (Gibco, USA) supplemented with $10 \%$ fetal bovine serum (FBS) (Gibco, USA), $10 \mu \mathrm{g} / \mathrm{ml}$ streptomycin sulfate and $100 \mu \mathrm{g} / \mathrm{ml}$ penicillin G. MG-63 cell lines were cultured in high-glucose Dulbecco's modified Eagle's medium (DMEM; Gibco, USA) supplemented with 10\% fetal bovine serum (FBS) (Gibco, USA), $10 \mu \mathrm{g} / \mathrm{ml}$ streptomycin sulfate and $100 \mu \mathrm{g} / \mathrm{ml}$ penicillin G. Cells were incubated at $37{ }^{\circ} \mathrm{C}$ in a humidified atmosphere containing $5 \% \mathrm{CO}_{2}$.

\section{RNA isolation and real-time PCR analysis}

Total RNA were extracted from fresh tissues and cells using TRIzol reagent (Invitrogen, CA, USA) according to the manufacturer's protocol. Total RNA (500 ng) was reverse-transcribed into complementary DNA using the Reverse Transcription Reagent Kit (TaKaRa, Japan). Realtime PCR analysis was performed using the 7500 RealTime PCR system (Applied Biosystems, USA) with a SYBR Green PCR Amplification Kit (TaKaRa). Primers are shown in Table 1. Each PCR analysis was performed in triplicate, and the results were normalized to $\beta$ actin expression. The $2^{-\Delta \Delta C t}$ method was used for data analysis.

\section{Western blot analysis}

Total proteins were extracted from fresh tissues and cells using RIPA Protein Lysis solution (Pierce, IL, USA) and quantified by the Bradford method. Prepared samples were electrophoresed by sodium dodecyl sulfate polyacrylamide gel electrophoresis and blotted onto a polyvinylidene fluoride membrane (Millipore, MA, USA) using the Tetra Handcast system (Bio-Rad, USA). The membrane was blocked for $1 \mathrm{~h}$ at room temperature or overnight at $4{ }^{\circ} \mathrm{C}$ in Tris-buffered saline with $0.05 \%$ Tween (TBST) containing 5\% non-fat milk. Then it was incubated overnight at $4{ }^{\circ} \mathrm{C}$ with the appropriate primary antibody, and followed by the secondary antibody for $70 \mathrm{~min}$ at room temperature. The protein bands were

Table 1 Primers used for the qRT-PCR analysis

\begin{tabular}{|c|c|c|}
\hline \multirow[t]{2}{*}{ genes } & \multicolumn{2}{|l|}{ Sequence $\left(5^{\prime}-3^{\prime}\right)$} \\
\hline & Forward primer & Reverse primer \\
\hline $50 \times 3$ & GACCTGTTCGAGAGAACTCATCA & CGGGAAGGGTAGGCTTATCAA \\
\hline E-cadherin & CGAGAGCTACACGTTCACGG & GGGTGTCGAGGGAAAAATAGG \\
\hline CK-18 & GGCATCCAGAACGAGAAGGAG & ATTGTCCACAGTATTTGCGAAGA \\
\hline $\mathrm{N}$-cadherin & TCAGGCGTCTGTAGAGGCTT & ATGCACATCCTTCGATAAGACTG \\
\hline Vimentin & GACGCCATCAACACCGAGTT & CTITGCGTTGGTTAGCTGGT \\
\hline Snail1 & AAGGCCTTCTCTAGGCCCT & CGCAGGTTGGAGCGGTCAG \\
\hline Snail2 & TTCGGACCCACACATTACCT & GCAGTGAGGGCAAGAAAAAG \\
\hline Twist1 & CAGCTACGCCTTCTCGGTCT & CTGTCCATITTCTCCTTCTCTGGA \\
\hline Zeb1 & GATGATGAATGCGAGTCAGATGC & ACAGCAGTGTCTTGTTGTTGT \\
\hline Zeb2 & CAAGAGGCGCAAACAAGCC & GGTTGGCAATACCGTCATCC \\
\hline Snail1 promoter & TCAGAAGCGCTCAGACCAC & TTATCTGCCACGCCCCITI \\
\hline Snail1 non-promoter & TGCTCATCTGGGACTCTGTC & GAGGAGAAGGACGAAGGAGC \\
\hline$\beta$ actin & TTGCCGACAGGATGCAGAAG & CAGCGAGGCCAGGATGGAGC \\
\hline
\end{tabular}


detected and quantified using the Gene Gnome Syngene Bio Imaging System (Syngene, UK) with an electrochemiluminescence kit (Pierce, IL, USA).

The primary anti-SOX3 antibody was purchased from Abcam (MA, USA), and the anti- $\beta$ actin antibody was purchased from Santa Cruz Biotechnology Inc. (CA, USA). Other primary antibodies (anti-E-cadherin, antiCK-18, anti-N-cadherin, anti-vimentin, anti-Snail1, antiTwist1, anti-Slug, anti-ZEB1, anti- ZEB2, anti-FLAG) were purchased from Cell Signaling Technology (MA, USA). Anti-mouse or anti-rabbit HRP-conjugated secondary antibodies were purchase from Santa Cruz Biotechnology Inc. (CA, USA).

\section{Lentivirus construction, Recombinant plasmid and siRNA transfection}

Lentivirus construction of SOX3-shRNA, control shRNA, SOX3-overexpression, and control-overexpression were purchased from Genechem Company (Shanghai, China). SOX3-targeting shRNA were designed: shRNA-1: 5'- GC ACATGAAGGAGTATCCGGA-3'; shRNA-2: 5'-GCCGT GCACATGAAGGAGTAT-3'; shRNA-3: 5'-GACGCTGC TCAAGAAAGATAA-3'; non-target control shRNA sequence: 5'-TTCTCCGAACGTGTCACGT-3'. Stable SO X3-knockdown and SOX3-upregulation were confirmed by quantitative real-time PCR and Western Blot analysis.

The plasmids expressed Snail1 and Snail1-siRNA were purchased from Genechem Company (Shanghai, China). The small interfering RNA (siRNA) sequence targeting Snail1 is as follows: 5'-GCGUGGGUUUUUGUAUCC $\mathrm{A}(\mathrm{dTdT})-3$ '. Cells were transfected with plasmid using Lipofectamine 2000 (Invitrogen) according to the manufacturer's protocol.

\section{Luciferase reporter assays}

The Snail1 promoter Luciferase reporter gene was generated from human genomic DNA corresponding to the sequence spanning $-2,340$ to +146 bp (relative to the transcriptional start site) of the 5'-flanking region of the human Snail1 gene. Various truncated reporter genes were generated as shown in Fig. 5. The constructs were confirmed by DNA sequencing. Cells were cultured in 24-well plates and transfected with 1ug reporter gene construct using Lipofectamine 2000 (Invitrogen) according to the manufacturer's instructions. After $24 \mathrm{~h}$ of transfection, luciferase activities were measured using the Dual-Glo ${ }^{\text {ma }}$ Luciferase Assay System kit (Promega, WI, USA). Luciferase intensities were normalized to the activity of Renilla luciferase.

\section{Chromatin immunoprecipitation assay}

We constructed two FLAG-tagged SOX3 variants harboring a deletion of the HMG domain or the C-terminal transcriptional activation domain (SOX3- $\triangle \mathrm{HMG}$ and SOX3- $\triangle$ CTD). After transfected with the plasmid for
$48 \mathrm{~h}$, OS cells were performed with Chromatin immunoprecipitation assay. OS cells were fixed with $1 \%$ formaldehyde and processed with the ChIP-IT Enzymatic kit (Active Motif, Carlsbad, CA) according to the manufacturer's instruction. The resulting protein/DNA complexes were then immuno-precipitated overnight at $4{ }^{\circ} \mathrm{C}$ using anti-Flag antibody (Roche, $\mathrm{CH}$ ), non-specific IgG antibodies (Santa Cruz Biotechnology Inc., CA, USA), respectively, with protein $\mathrm{G}$ magnetic beads. After eluted from beads and incubated with proteinase $K$, the precipitated DNA were amplified and quantified using PCRs. The primers for Snail1 promoter are shown in Table 1.

\section{Cell migration and matrigel invasion assays}

Cell migration was evaluated using the wound-healing migration assays. Cells were cultured to form a tight cell monolayer and then wounded with $10 \mu \mathrm{L}$ plastic pipette tip. The remaining cells were washed twice with culture medium to remove cell debris and cultured in normal serum-containing culture medium with the presence of Mitomycin C (Amresco) at the concentration of $10 \mu \mathrm{g} / \mathrm{ml}$. The incubation is at $37{ }^{\circ} \mathrm{C}$ with humidified atmosphere containing $5 \% \mathrm{CO} 2$. At the indicated times, migrating cells at the wound front were photographed. Migration capacity was quantitated by measuring the percent of open area in 3-5 randomly captured images.

Cell invasion assays were performed using 24-well Transwell chambers $(8 \mu \mathrm{m}$ pore size; Millipore) precoated with 100 ul Matrigel (1:7 dilution; BD Biosciences, San Jose, CA, USA). In total, $1 \times 10^{5}$ cells were suspended in $100 \mu \mathrm{L}$ serum-free medium with the presence of mitomycin $C(10 \mathrm{ug} / \mathrm{ml})$ and were added to the upper chamber, and $600 \mu \mathrm{L}$ DMEM that contained $10 \%$ FBS were placed in the lower chamber. After $24 \mathrm{~h}$ of incubation, Matrigel and the cells remaining in the upper chamber were removed by cotton swabs. Cells on the lower surface of the membrane were fixed in $4 \%$ paraformaldehyde and stained with crystal violet. Cells at $\times 200$ magnification were counted and photographed. All experiments were performed in triplicate.

\section{In vivo lung metastasis model}

The single-cell suspensions of $5 \times 10^{6}$ OS cells $/ 0.5 \mathrm{ml}$ were injected into the tail vein of 5 -week-old female Nude mice. The mice were sacrificed at 56 days after injection. The lungs were resected, and measured by fluorescent areas measured using the Image-Pro Plus software 6.0 (Media Cybernetics Inc., Silver Spring, MD, USA).

\section{Immunohistochemistry analysis}

Immunohistochemical staining was performed according to the method of our previous studies [26]. Typically, after rehydration and antigen retrieval, the slides were incubated with primary antibodies against SOX3 (1:100, 
Abcam, MA, USA), Snail1(1:100, Cell Signaling Technology, MA, USA) and E-cadherin (1:100, Cell Signaling Technology, MA, USA) at $4{ }^{\circ} \mathrm{C}$ overnight, followed by HRP-conjugated secondary antibody at $37^{\circ} \mathrm{C}$ for $30 \mathrm{~min}$. Then the slides were stained with 3,3'-diaminobenzidine (DAB) and Mayer's hematoxylin. Immunoreactivity Score was conducted by two independent observers, who were blinded to the patients' information. Five high-power fields (magnification, $400 \times$ ) were randomly selected. Based on the intensity of positive staining, immunoreactivity was categorized as follows: negative $(-)$, weak $(+-)$, moderate $(+)$, strong $(++)$. Negative and weak correspond to low expression, moderate and strong correspond to high expression.

\section{Statistical analysis}

Statistical analysis was performed using SPSS16.0 statistical software (SPSS Inc., Chicago, IL, USA). Student's $t$ tests were used to assess significant differences among study groups. The level of significance was set at $p<0.05$. All experiments were performed at least in triplicate.

\section{Results}

\section{SOX3 is upregulated in human OS tissues}

We first determined the SOX3 mRNA and protein levels in 42 cases of human OS tissues and in adjacent nontumor samples by qRT-PCR and Western blot analysis. SOX3 expression was increased in most OS samples relative to non-tumor samples (Fig. 1a, b). Next, the SOX3 mRNA level was assessed in OS tissues and bone cysts (Fig. 1c). SOX3 was overexpressed in most OS tissues compared with that in bone cysts. We further evaluated SOX3 expression in various OS cell lines with different metastatic potential (U2OS, SoSP-M, SoSP9607, and MG-63) and the immortal normal osteoblast cell line, hFOB1.19. SOX3 was expressed at a relatively higher level in all four OS cell lines compared with hFOB1.19 (Fig. 1d), suggesting that SOX3 is upregulated in OS cell lines.

\section{SOX3 promotes OS cell migration and invasion}

To explore the role of SOX3 in OS progression, we examined the effects of SOX3 overexpression or knockdown on the migratory and invasive abilities of OS cells. The expression of SOX3 is relatively low in MG63 and high in U2OS cells. Therefore, we conducted stable SOX3 overexpression in MG63 cells (MG63-SOX3) and successful knockdown of SOX3 in U2OS cells (U2OSsh-SOX3) (Fig. 2a). Wound-healing migration assays revealed that MG63-SOX3 cells had an increased rate of wound closure compared with the MG63-control, and that U2OS-sh-SOX3 cells had delayed wound closure compared with the U2OS-sh-control (Fig. 2b). Transwell $^{\circ}$ Matrigel $^{\circ}$ assays showed that the invasive MG63-
SOX3 cell counts were significantly higher than that of the control cells and that the invasive U2OS-shSOX3 cell number was decreased compared with the control (Fig. 2c).

The lung is the most common site of OS metastasis. Therefore, we used a xenograft nude mouse model to examine the role of SOX3 expression in lung metastasis in vivo. We first injected MG63-control and MG63-SOX3 cells into nude mice via the tail vein. After 8 weeks, obvious lung metastases were detected in the lungs of recipient mice in the MG63-SOX3 group compared with the MG63-control group. We then injected U2OS-sh-control and U2OS-sh-SOX3 cells following the same protocol. As expected, knockdown of SOX3 significantly reduced lung metastasis in U2OS-sh-SOX3 cells compared with the U2OS-sh-control group (Fig. 2d). Therefore, SOX3 appears to play an oncogenic role in OS progression.

\section{SOX3 induces EMT and upregulates Snail1 expression in OS cells}

EMT is the critical process and mechanism by which local invasion and distant metastasis are initiated. Overexpression of SOX3 in MG63 cells led to significantly higher levels of mesenchymal markers $(\mathrm{N}$-cadherin and vimentin) and lower levels of epithelial markers (E-cadherin and Keratin 18) (Fig. 3a, c), whereas knockdown of SOX3 in U2OS cells caused increased expression of epithelial markers and decreased expression of mesenchymal markers (Fig. 3a, c). These data confirm that SOX3 induces EMT in OS cells.

To further define the mechanism by which SOX3 regulates EMT, we determined whether SOX3 modulates the expression of several known EMT-related transcription factors, including Zeb1, Zeb2, Snail1, Slug, and Twist1. We determined that the alterations in SOX3 expression led to significant changes in the expression of Snail1 (Fig. 3b, c), indicating that SOX3 may induce EMT by enhancing Snail1 expression.

\section{SOX3-promoted EMT is mediated by Snail1}

To confirm the involvement of Snail1 in the SOX3induced EMT, siRNA targeting Snail1 (siSnail1) was transfected into MG63-SOX3 cells. siSnail1 partially abolished the increased migratory and invasive abilities induced by SOX3 overexpression (Fig. 4a), whereas upregulation of Snail1 in U2OS-shSOX3 cells transfected with a Snail1 plasmid (Snail1) rescued the decreased cell migration and invasion induced by SOX3 knockdown (Fig. 4b). Additionally, qRT-PCR and Western blot analysis demonstrated that the alterations in EMT markers by SOX3 were reversed by siSnail1 or the Snail1 plasmid (Fig. 4c, d). 

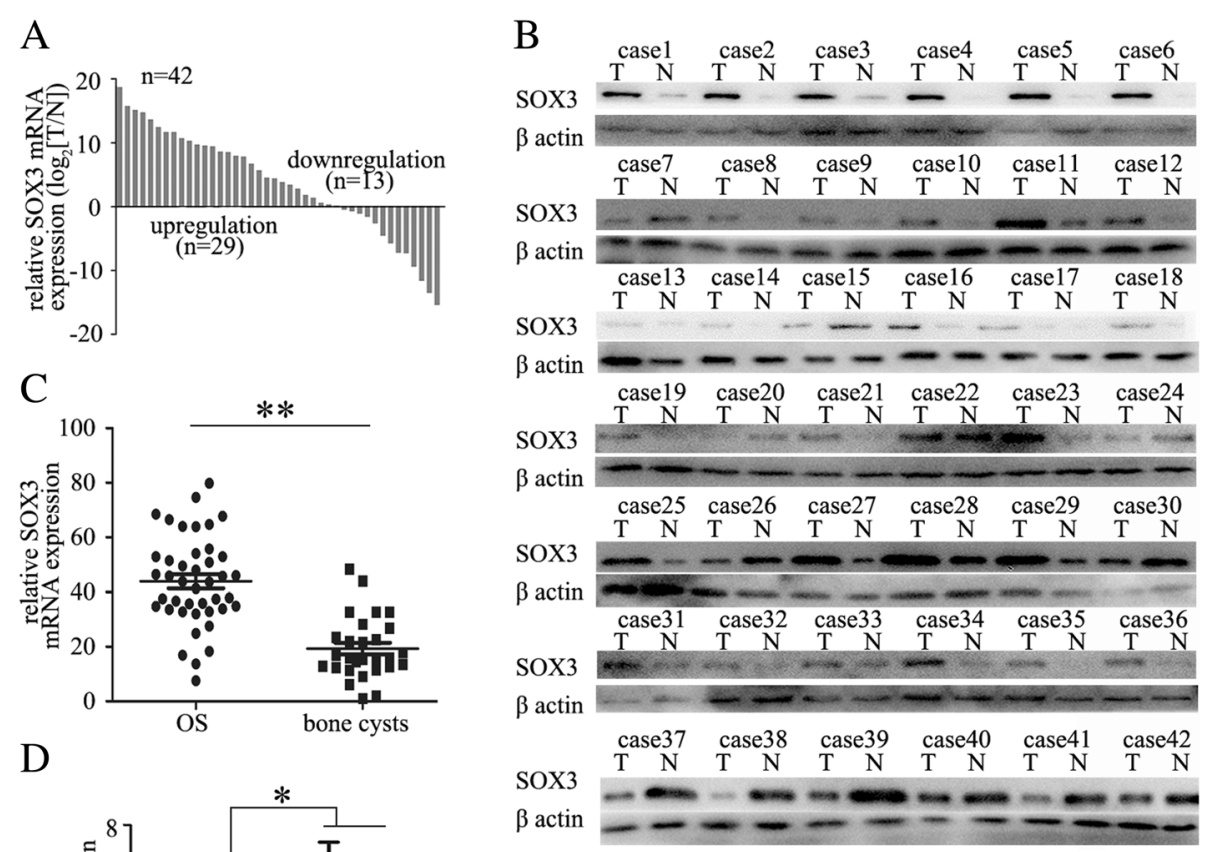

$\mathrm{D}$
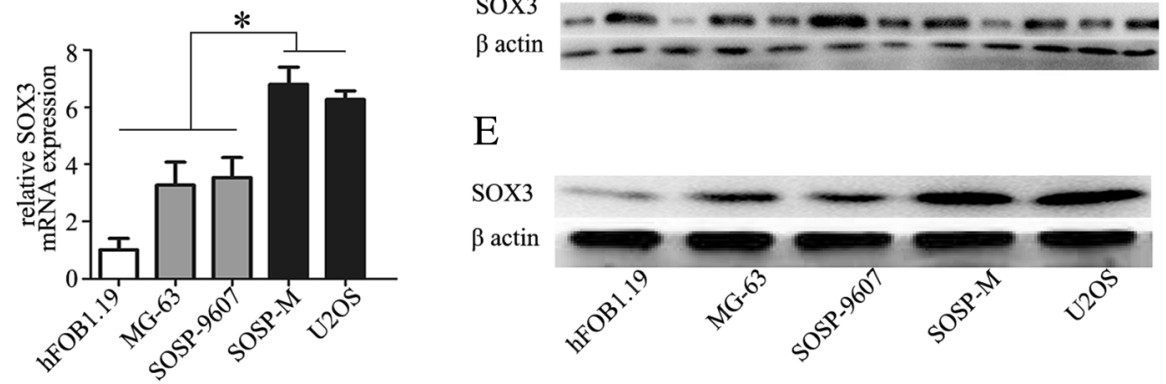

Fig. 1 SOX3 is upregulated in human osteosarcoma tissues. a, b Relative SOX3 mRNA and protein levels were significantly increased in osteosarcoma tissues when compared with the adjacent non-tumor tissues $(n=42)$. c Relative SOX3 mRNA level was significantly higher in osteosarcoma tissues $(n=42)$ than that in bone cysts $(n=28)$. $\mathbf{d}$, e SOX3 mRNA and protein levels in different OS cell lines. Data represent the mean \pm SD of triplicate samples; ${ }^{*} P<0.05,{ }^{* *} P<0.01$

\section{SOX3 promotes Snail1 transcription via binding to its promoter region}

To explore the mechanism by which SOX3 regulates Snail1 expression in OS cells, we assessed the SOX3dependent regulation of Snail1 mRNA transcription. The promoter region spanning $-2,340$ to $+146 \mathrm{bp}$ of Snail1 was used in a luciferase reporter assay. MG63control and MG63-SOX3 cells were transfected with the reporter gene. SOX3 overexpression in MG63 cells promoted the reporter activity of Snail1 (Fig. 5c). Sequence analyses of the Snail1 promoter region showed three putative SOX3-binding sites (Fig. 5a, b). To determine the cis-regulatory elements of the Snail 1 promoter in response to SOX3 regulation, various truncated reporter genes were constructed (Fig. 5c). SOX3 overexpression affected the transactivation of the shortest construct containing only the third binding site promoter sequence ( -651 to $+146 \mathrm{bp}$ ), while the deletion of sites 1 and 2 had no significant effect on SOX3-regulated Snail1 promoter activity. The deletion or substitution of site 3 showed notable inhibition of Snail1 promoter activity, suggesting that site 3 is the critical core binding site of SOX3 (Fig. 5c).

SOX3 contains a DNA-binding HMG domain and a $\mathrm{C}$-terminal transcriptional activation domain. To determine the region required for its ability to transactivate Snail1 mRNA, we constructed two FLAG-tagged SOX3 variants harboring a deletion of the HMG domain or the C-terminal transcriptional activation domain (SOX3$\triangle$ HMG and SOX3- $\triangle \mathrm{CTD}$, respectively) and performed a chromatin immunoprecipitation (ChIP) assay. SOX3WT and SOX3- $\triangle$ CTD, but not SOX3- $\triangle$ HMG, pulled down the promoter region but not the non-promoter region of the Snail1 gene (Fig. 5d, e). These data demonstrate that the HMG domain is critical for SOX3activated Snail1 expression.

\section{SOX3 expression correlates with that of Snail1 and E- cadherin in human OS tissues}

We next assessed the relationships between SOX3, Snail1, and E-cadherin expression by immunohistochemistry in 18 human OS tissues. Twelve OS tissues exhibited high 


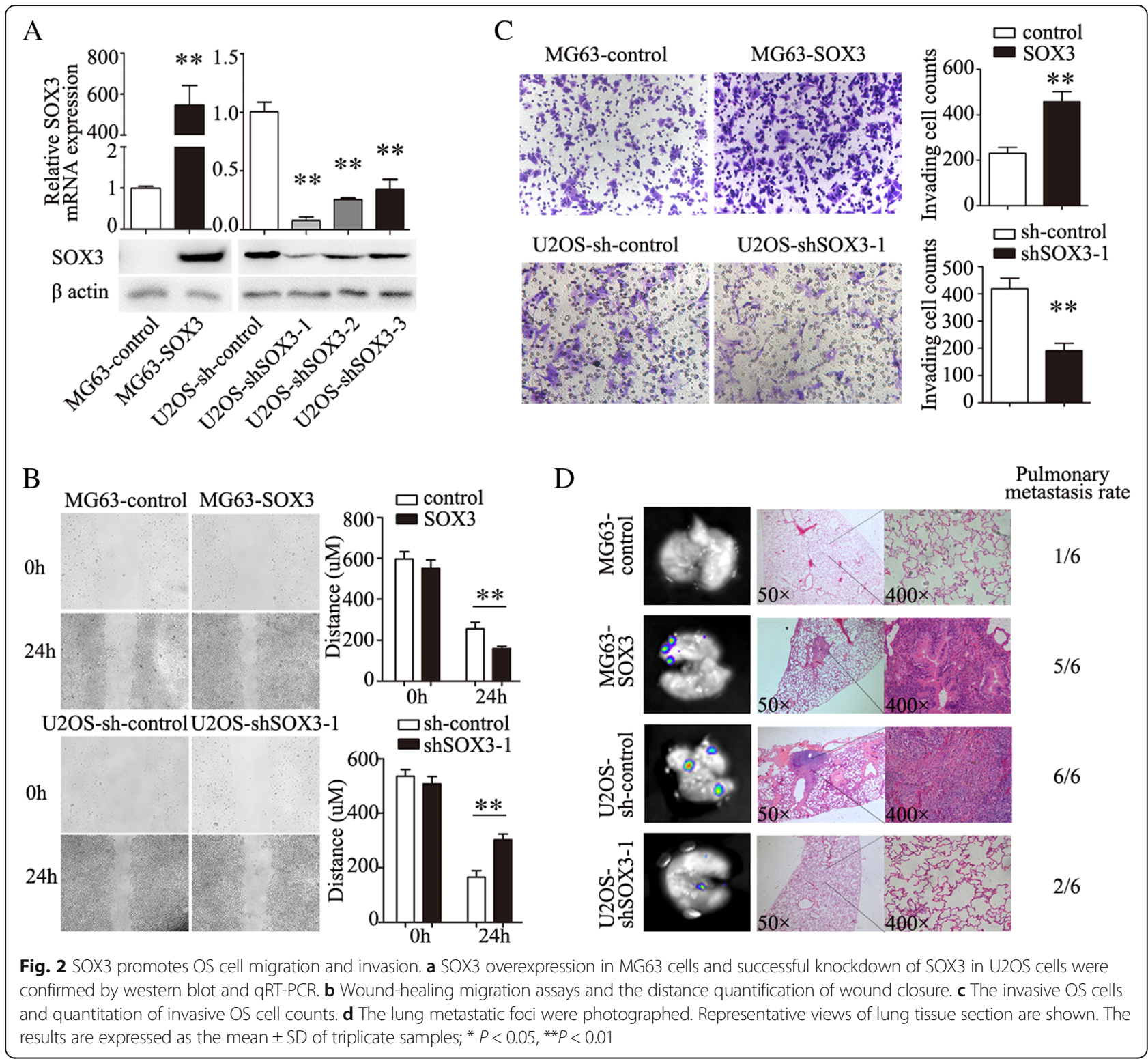

expression of SOX3; 6 had low expression. Nine of 12 tumors with high SOX3 expression tended to have higher Snail1 levels and seven showed lower E-cadherin levels (Table 2). In contrast, 4 of 6 tumors with low SOX3 expression exhibited lower Snail1 expression and 5 exhibited higher E-cadherin expression (Table 2). These data suggest that SOX3 expression positively correlates with that of Snaill and negatively correlates with E-cadherin (Fig. 6a). Additionally, correlation analysis between the mRNA expression of SOX3, Snail1, and E-cadherin in 42 OS tissues also show the similar results (Fig. 6b). We also analyzed the relationship between SOX3 and the clinical pathological characteristic (Table 2). However, there were no significant statistically.

\section{Discussion}

SOX3 is a member of the SOX family of transcription factors that plays important roles in the regulation of embryonic development and in the determination of cell fate $[17,19,20]$. Recent studies have reported that SOX3 is linked to various cancers, including esophageal squamous cell carcinoma, ovarian cancer, and T-cell lymphomas $[22,23,25]$. In the present study, we first demonstrated that SOX3 was expressed at a higher level in OS tissues compared with normal tissues. In vivo and in vitro experiments suggested that SOX3 promoted OS cell invasion and metastasis. Further analysis showed that SOX3 significantly induced EMT by promoting Snail1 expression. Moreover, a positive correlation between SOX3 and Snail1 

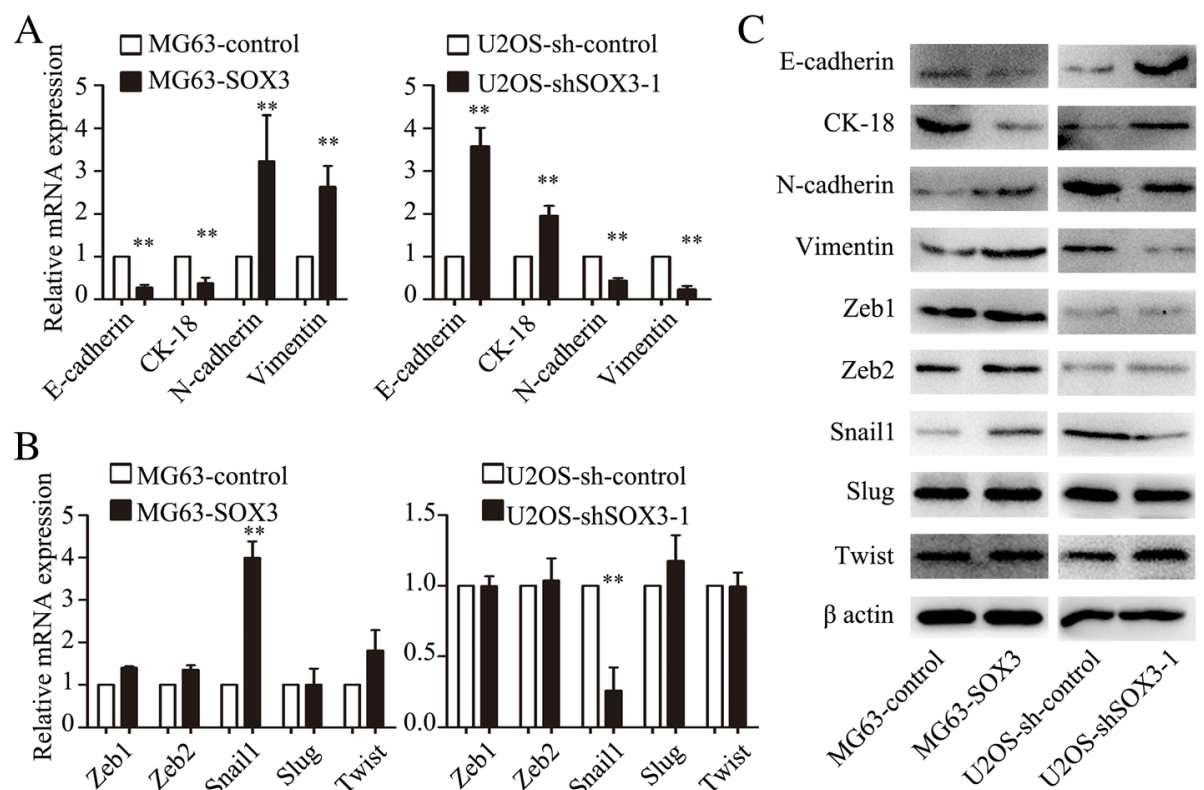

Fig. 3 SOX3 induces EMT and upregulates Snail1 expression. a qRT-PCR shows changes of EMT marker expression affected by SOX3 upregulation and downregulation. $\mathbf{b}$ qRT-PCR shows changes of EMT-related transcription factors expression affected by SOX3 upregulation and downregulation. c western blot shows the protein expression of EMT markers and related transcription factors. The results are expressed as the mean \pm SD of triplicate samples; ${ }^{*} P<0.05,{ }^{* *} P<0.01$

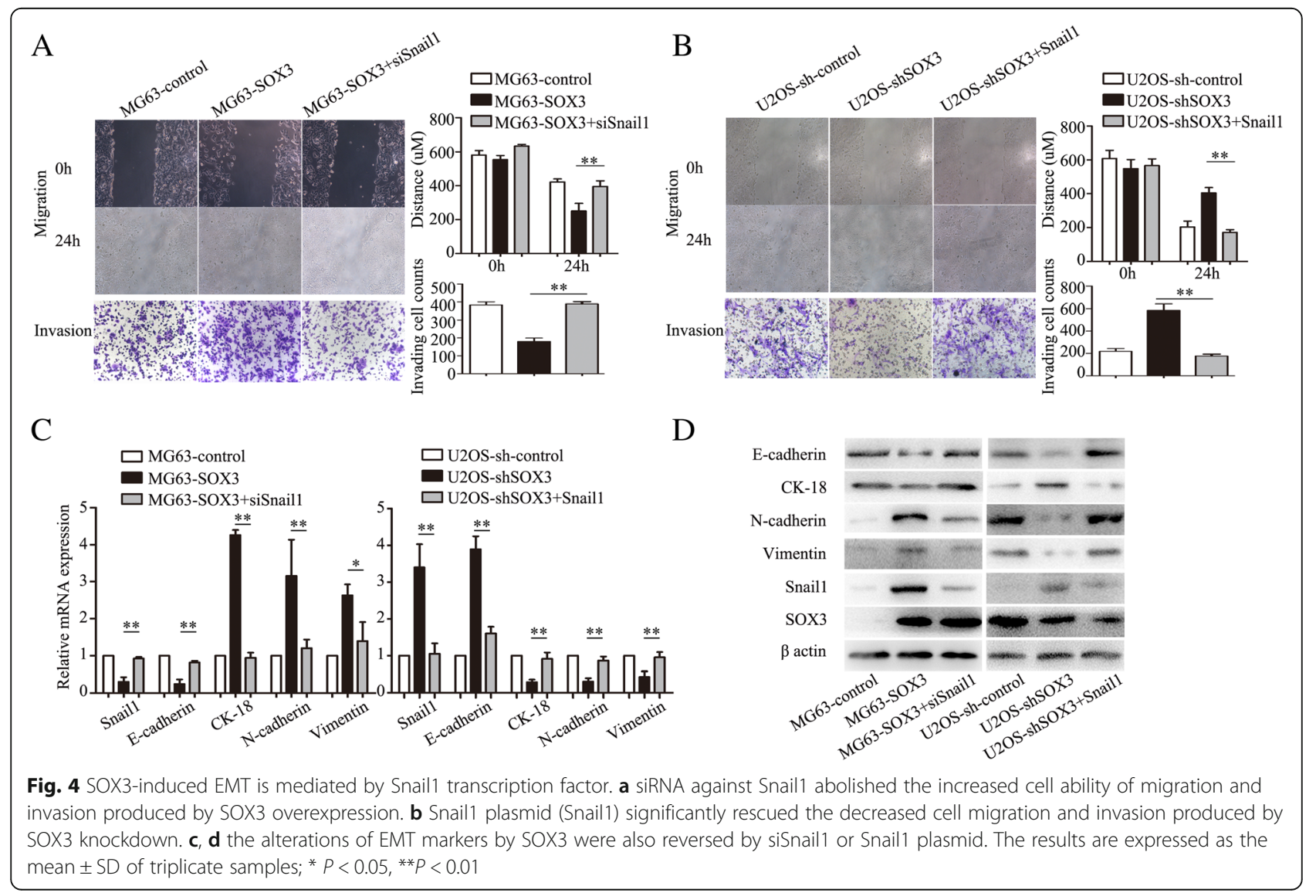


A

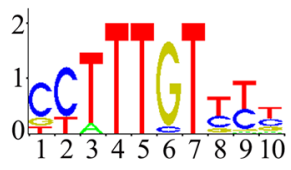

B

- Three putative core binding sites site 1: tccttcatTCTTTGTTTCagcagcaac site2: cccatgcctTCTTTCTCCC gccagccta site3: gccacgcc CCTTTGTCACctccgcgcca site3 deletion: gccacgec----------ctcegegeca site3 substitution: gecaegce $A A C T C A C G T G \mathrm{ctc}$ gegeca
$\mathrm{C}$

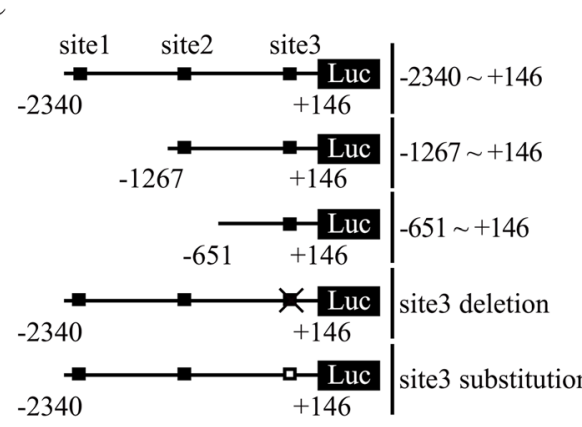

Folds of Luciferase activity

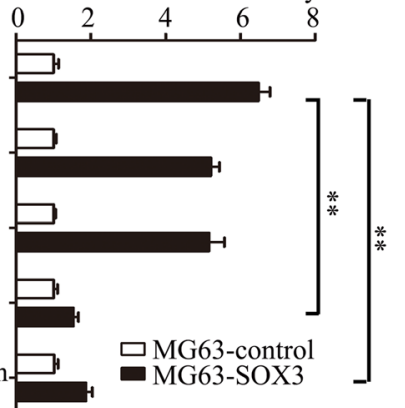

$\mathrm{D}$

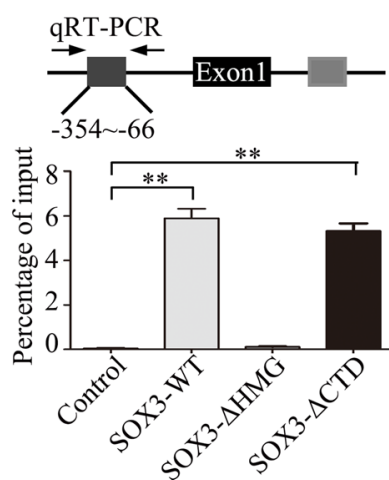

E
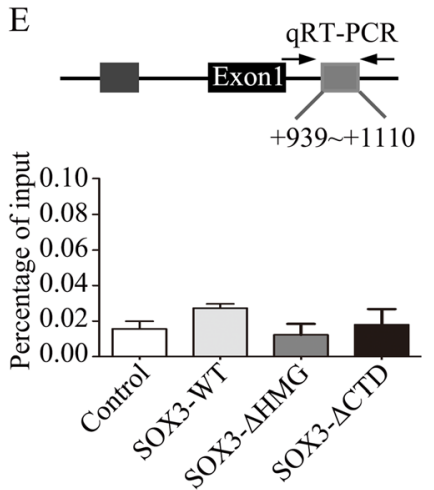

Fig. 5 SOX3 promotes Snail1 transcription via binding to the promoter region. a Sequence logo of SOX3 from JASPAR database. b Three putative SOX3-binding sites in Snail1 promoter region. c Truncation analysis of the Snail1 reporters. Five kinds of reporters, including upstream regions from bp $-2340,-1267,-651$ to $b p+146$, and upstream regions with site 3 deletion or site 3 substitution, were analyzed for their activity. (d, e) FLAG-tagged SOX3 variants (SOX3- $\Delta \mathrm{HMG}$ and SOX3- $\Delta$ CTD) were followed by chromatin immunoprecipitation (ChIP) assay. Precipitated DNAs were quantified by qRT-PCR for promoter and non-promoter regions of Snail 1 gene. The results are expressed as the mean \pm SD of triplicate samples; ${ }^{*} P<0.05,{ }^{*} P<0.01$

expression was validated in OS samples. These data suggest that SOX3 plays a crucial role in OS progression.

Cancer has been proposed to have six fundamental hallmarks [27], which include self-sufficiency in growth factors, insensitivity to growth-inhibitory signals, evasion of apoptosis, limitless replicative potential, sustained angiogenesis, and the ability to invade and metastasize. The primary tumor is rarely fatal to the organism. It is the final hallmark of cancer, invasion and metastasis, which underlies its deadly progressive nature [27]. Metastatic spread is responsible for more than $90 \%$ of all cancer-related deaths, yet, despite this being well-known, metastasis in and of itself remains the most poorly understood component of cancer progression. OS is also characterized by a high propensity for metastasis (especially to the lung), which is the leading cause of death [1]. Our study revealed a crucial role for
SOX3 in promoting OS invasion and metastasis both in vitro and in vivo, strongly suggesting that SOX3 may serve as a therapeutic target for patients with metastatic OS.

EMT is a key event in the tumor invasion and metastasis process that enables epithelial cells to acquire an invasive mesenchymal phenotype. EMT is characterized by a loss of intercellular adhesion (E-cadherin), downregulation of epithelial makers (cytokeratins), upregulation of mesenchymal markers (vimentin), acquisition of a spindle-like morphology, and an increase in motility, invasiveness, and metastatic capabilities [6]. In addition, the process known as "cadherin switching" (downregulation of E-cadherin and upregulation of mesenchymal cadherins such as $\mathrm{N}$ cadherin [28]) and the accumulation of $\beta$-catenin have also been associated with EMT [29]. Previous studies have revealed the increased EMT characteristics in OS cells and 
Table 2 The relationship between SOX3 expression and clinicopathological features OS

\begin{tabular}{lllll}
\hline Clinicopathological features & \multicolumn{2}{l}{ SOX3 } & P value \\
\cline { 3 - 5 } & & Low & High & \\
\hline Age (y) & $\leq 20$ & 3 & 8 & 0.494125 \\
Gender & $>20$ & 3 & 4 & \\
\multirow{2}{*}{ Grade } & Male & 4 & 7 & 0.73244 \\
& Female & 2 & 5 & \\
\multirow{2}{*}{ Metastasis } & Grade1 & 2 & 2 & 0.637628 \\
& Grade2 & 3 & 6 & \\
5-year survival & Grade3 & 1 & 4 & \\
& Yes & 2 & 6 & 0.502335 \\
Snail1 & No & 4 & 6 & \\
& Absent & 1 & 5 & 0.288844 \\
E-cadherin & Present & 5 & 7 & \\
& Low & 4 & 3 & 0.087375 \\
& High & 2 & 9 & \\
\hline
\end{tabular}

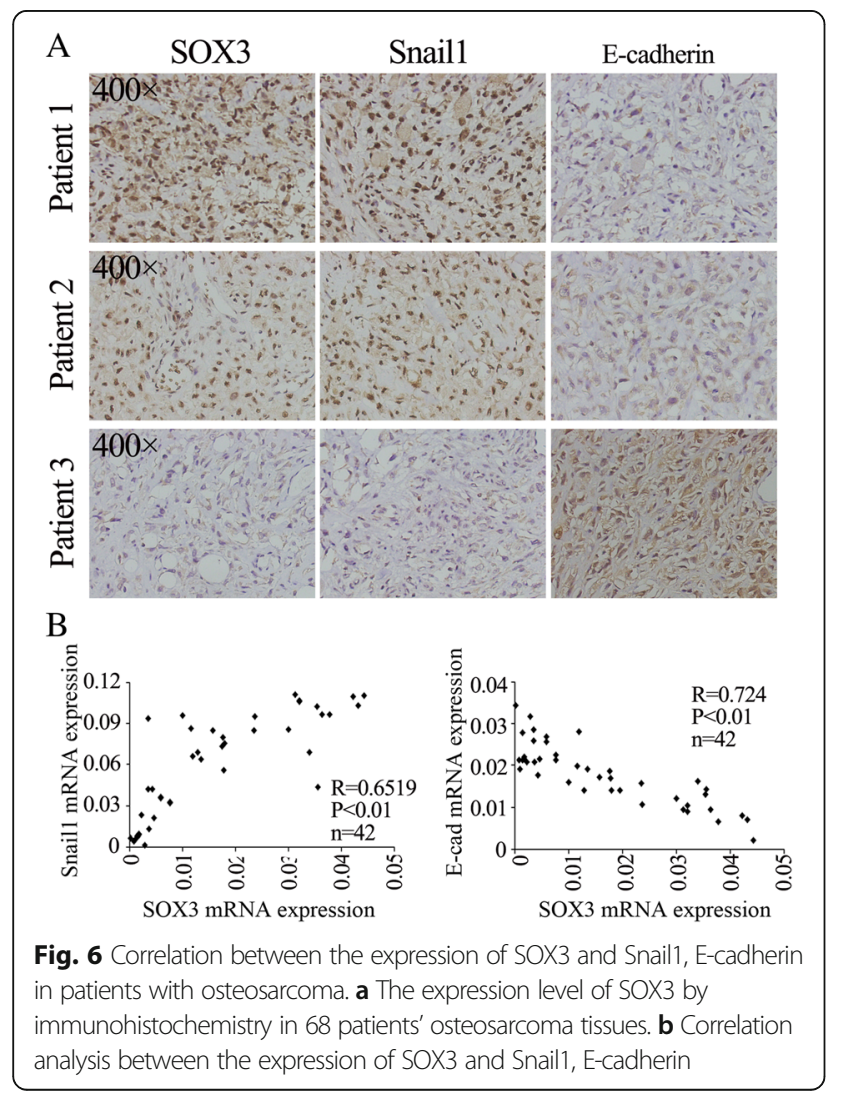

demonstrated the involvement of EMT in OS invasiveness and migration [30-33]. In our study, the decreased invasive ability of the U2OS cell line induced by the depletion of SOX3 prompted us to investigate whether SOX3 is involved in EMT. As expected, SOX3 silencing upregulated epithelial markers, such as E-cadherin, and downregulated mesenchymal markers, such as $\mathrm{N}$-cadherin and vimentin. Consistent with these data, ectopic expression of SOX3 in MG63 cells led to opposing results. This involvement of SOX3 in regulating EMT provided an explanation for the increased levels of SOX3 expression promoting OS cell invasiveness and metastasis.

EMT is a very complex process that is executed in response to pleiotropic signaling factors that include several transcriptional repressors, such as Snail1, Snail2, Zeb1, Zeb2, and Twist. The roles of these factors in promoting invasiveness and metastasis have been widely reported [34-38]. However, the upstream mechanism of these transcriptional repressors in cancer progression are still elusive. Previous studies revealed that SOX family of proteins play important role in cancer development [39-41]. For example, SOX5 promoted EMT process by regulation of Twist1 in hepatocellular carcinoma [41]. These literatures give us an implication that SOX3, as a member of SOX family, might play the potential role in regulating EMTrelated transcriptional factors. Therefore, our study investigated the hypothesis and demonstrated that SOX3 enhanced Snail1 expression via binding to the site 3 region of the Snail1 promoter, thereby inducing EMT and promoting OS cell invasion and metastasis.

Previous studies have reported that SOX3 is correlated with the development of several types of cancer, including esophageal squamous cell carcinoma, ovarian cancer, and T-cell lymphomas, and induces oncogenic transformation of chicken embryonic fibroblasts [22, 23, 25]. These findings, together with our results showing that SOX3 is dramatically overexpressed in OS tissues and promotes OS cell invasion and metastasis, suggest that SOX3 may act as an oncogene. However, before defining SOX3 as an oncogene in OS progression, the relationship between SOX3 expression and the prognosis, pathology, and clinical phenotype of patients with OS should be investigated. Our studies showed that there was no significant relationship between SOX3 expression and the clinical pathological features in 18 patients with OS. This might be related to the limited sample size. The collection of a larger number of samples and clinical information is currently underway.

\section{Conclusion}

In conclusion, the collective findings from our study show for the first time that SOX3 acts as a metastasis-associated gene in OS. The mechanistic link among SOX3, Snail1, and EMT indicates SOX3 as a potential therapeutic target for OS metastasis. 


\section{Abbreviations}

ChIP: Chromatin immunoprecipitation; CK-18: Keratin 18; CSC: Cancer stem cell; EMT: Epithelial-mesenchymal transition; EMT-TFs: Transcription factors associated with EMT; OS: Osteosarcoma; GRT-PCR: Quantitative real-time polymerase chain reaction; siSnail1: siRNA targeting Snail1; SOX3: Sexdetermining region $\mathrm{Y}$-box protein 3

\section{Acknowledgements}

Not applicable.

\section{Funding}

This study was sponsored by grants from the National Natural Science Foundation of China (No. 81271998) and the National High-Tech Research and development program (863-Project, No. 2015AA020316).

\section{Availability of data and materials}

Not applicable.

\section{Authors' contributions}

$\mathrm{YH}$ and $\mathrm{MQ}$ conceived and designed the study. MQ, DC, CS performed the experiments. JS, $\mathrm{HZ}$ analyzed the data. All authors read and approved the final manuscript.

\section{Competing interests}

All authors have no conflicts of interest to disclose.

\section{Consent for publication}

Informed consent for publication was obtained from all subjects or their guardians.

\section{Ethics approval and consent to participate}

Ethical approval for study was provided by the ethics committee of Shanghai Jiao Tong University Affiliated Sixth People's Hospital. Written informed consent was obtained from all subjects or their guardians.

\section{Publisher's Note}

Springer Nature remains neutral with regard to jurisdictional claims in published maps and institutional affiliations.

\section{Author details}

'Department of Sports Medicine, Shanghai Jiao Tong University Affiliated Sixth People's Hospital, 600 Yishan Road, Shanghai 200233, China. ${ }^{2}$ Department of Gastrointestinal Surgery, West China Hospital, Sichuan University, Chengdu 610041, Sichuan, China.

\section{Received: 18 January 2017 Accepted: 11 March 2017} Published online: 23 March 2017

\section{References}

1. Jaffe N. Osteosarcoma: review of the past, impact on the future, The American experience. Cancer Treat Res. 2009;152:239-62.

2. Palmerini E, Fabbri N, Staals EL, Marchesi E, Alberghini M, Tienghi A, Fagioli F, Picci P, Mercuri M, Ferrari S. Osteosarcoma of the pelvis and sacrum: a retrospective analysis of 73 patients. J Clin Oncol. 2011;29(15_suppl):10039.

3. Hegyi M, Semsei AF, Jakab Z, Antal I, Kiss J, Szendroi M, Csoka M, Kovacs G. Good prognosis of localized osteosarcoma in young patients treated with limb-salvage surgery and chemotherapy. Pediatr Blood Cancer. 2011;57(3): 415-22.

4. Mialou V, Philip T, Kalifa C, Perol D, Gentet JC, Marec-Berard P, Pacquement $\mathrm{H}$, Chastagner P, Defaschelles AS, Hartmann O. Metastatic osteosarcoma at diagnosis: prognostic factors and long-term outcome-the French pediatric experience. Cancer Am Cancer Soc. 2005;104(5):1100-9.

5. Zavadil J, Haley J, Kalluri R, Muthuswamy SK, Thompson E. Epithelialmesenchymal transition. Cancer Res. 2008;68(23):9574-7.

6. Thiery JP, Acloque H, Huang RY, Nieto MA. Epithelial-mesenchymal transitions in development and disease. Cell. 2009:139(5):871-90.

7. Lee JM, Dedhar S, Kalluri R, Thompson EW. The epithelial-mesenchymal transition: new insights in signaling, development, and disease. J Cell Biol. 2006;172(7):973-81.

8. Larsen JE, Nathan V, Osborne JK, Farrow RK, Deb D, Sullivan JP, Dospoy PD, Augustyn A, Hight SK, Sato M, Girard L, Behrens C, Wistuba II, Gazdar AF,
Hayward NK, Minna JD. ZEB1 drives epithelial-to-mesenchymal transition in lung cancer. J Clin Invest. 2016;126(9):3219-35.

9. Gao Y, Xuan XY, Zhang HY, Wang F, Zeng QR, Wang ZQ, Li SS. Relationship between TWIST expression and epithelial-mesenchymal transition of oesophageal squamous cell carcinoma. Cell Biol Int. 2012;36(6):571-7.

10. Kalluri $R$, Weinberg RA. The basics of epithelial-mesenchymal transition. J Clin Invest. 2009;119(6):1420-8.

11. Ru N, Liang J, Zhang F, Wu W, Wang F, Liu X, Du Y. SPRY4 intronic transcript 1 promotes Epithelial-mesenchymal transition through association with Snail1 in Osteosarcoma. DNA Cell Biol. 2016;35(6):290-5.

12. Robson EJ, Khaled WT, Abell K, Watson CJ. Epithelial-to-mesenchymal transition confers resistance to apoptosis in three murine mammary epithelial cell lines. Differentiation. 2006;74(5):254-64

13. Vega S, Morales AV, Ocana OH, Valdes F, Fabregat I, Nieto MA. Snail blocks the cell cycle and confers resistance to cell death. Genes Dev. 2004;18(10): 1131-43.

14. Zeisberg M, Neilson EG. Biomarkers for epithelial-mesenchymal transitions. J Clin Invest. 2009;119(6):1429-37.

15. Canesin G, Cuevas EP, Santos V, Lopez-Menendez C, Moreno-Bueno G, Huang Y, Csiszar K, Portillo F, Peinado H, Lyden D, Cano A. Lysyl oxidase-like 2 (LOXL2) and E47 EMT factor: novel partners in E-cadherin repression and early metastasis colonization. Oncogene. 2015;34(8):951-64.

16. Vidal B, Santella A, Serrano-Saiz E, Bao Z, Chuang CF, Hobert O. C. elegans SoxB genes are dispensable for embryonic neurogenesis but required for terminal differentiation of specific neuron types. Development. 2015;142(14): 2464-77.

17. Vetro A, Dehghani MR, Kraoua L, Giorda R, Beri S, Cardarelli L, Merico M, Manolakos E, Parada-Bustamante A, Castro A, Radi O, Camerino G, Brusco A, Sabaghian M, Sofocleous C, Forzano F, et al. Testis development in the absence of SRY: chromosomal rearrangements at SOX9 and SOX3. Eur J Hum Genet. 2015;23(8):1025-32

18. Bell DM, Leung KK, Wheatley SC, Ng LJ, Zhou S, Ling KW, Sham MH, Koopman P, Tam PP, Cheah KS. SOX9 directly regulates the type-II collagen gene. Nat Genet. 1997;16(2):174-8.

19. Fu L, Shi YB. The Sox transcriptional factors: Functions during intestinal development in vertebrates. Semin Cell Dev Biol. 2016.

20. Yin C. Molecular mechanisms of Sox transcription factors during the development of liver, bile duct, and pancreas. Semin Cell Dev Biol. 2017;63: 68-78.

21. Uwanogho D, Rex M, Cartwright EJ, Pearl G, Healy C, Scotting PJ and Sharpe PT. Embryonic expression of the chicken Sox2, Sox3 and Sox11 genes suggests an interactive role in neuronal development. Mech Dev. 1995:49:23-36.

22. Li K, Wang RW, Jiang YG, Zou YB, Guo W. Overexpression of Sox3 is associated with diminished prognosis in esophageal squamous cell carcinoma. Ann Surg Oncol. 2013;20 Suppl 3:S459-66.

23. Yan $Q$, Wang $F$, Miao $Y$, Wu X, Bai M, Xi X, Feng $Y$. Sex-determining region Y-box3 (SOX3) functions as an oncogene in promoting epithelial ovarian cancer by targeting Src kinase. Tumour Biol. 2016:37(9):12263-71.

24. Kim R, Trubetskoy A, Suzuki T, Jenkins NA, Copeland NG, Lenz J. Genomebased identification of cancer genes by proviral tagging in mouse retrovirus-induced T-cell lymphomas. J Virol. 2003;77(3):2056-62.

25. Xia Y, Papalopulu N, Vogt PK, Li J. The oncogenic potential of the high mobility group box protein Sox3. Cancer Res. 2000;60(22):6303-6.

26. Dong $Y$, Shen $X$, He M, Wu Z, Zheng Q, Wang Y, Chen Y, Wu S, Cui J, Zeng $Z$. Activation of the JNK-C-Jun pathway in response to irradiation facilitates Fas ligand secretion in hepatoma cells and increases hepatocyte injury. J Exp Clin Cancer Res. 2016;35(1):114.

27. Hanahan D, Weinberg RA. Hallmarks of cancer: the next generation. Cell. 2011;144(5):646-74.

28. Yao D, Dai C, Peng S. Mechanism of the mesenchymal-epithelial transition and its relationship with metastatic tumor formation. Mol Cancer Res. 2011; 9(12):1608-20

29. Gheldof A, Berx G. Cadherins and epithelial-to-mesenchymal transition. Prog Mol Biol Transl Sci. 2013;116:317-36.

30. Xu G, Guo Y, Xu D, Wang Y, Shen Y, Wang F, Lv Y, Song F, Jiang D, Zhang $Y$, Lou $Y$, Meng $Y$, Yang $Y$, Kang $Y$. TRIM14 regulates cell proliferation and invasion in osteosarcoma via promotion of the AKT signaling pathway. Sci Rep. 2017;7:42411.

31. Lv YF, Dai H, Yan GN, Meng G, Zhang X, Guo QN. Downregulation of tumor suppressing STF CDNA 3 promotes epithelial-mesenchymal transition and 
tumor metastasis of osteosarcoma by the Wnt/GSK-3beta/beta-catenin/Snail signaling pathway. Cancer Lett. 2016;373(2):164-73.

32. Guo X, Zhang J, Pang J, He S, Li G, Chong Y, Li C, Jiao Z, Zhang S, Shao M. MicroRNA-503 represses epithelial-mesenchymal transition and inhibits metastasis of osteosarcoma by targeting c-myb. Tumour Biol. 2016;37(7):9181-7.

33. Liu X, Liang Z, Gao K, Li H, Zhao G, Wang S, Fang J. MicroRNA-128 inhibits EMT of human osteosarcoma cells by directly targeting integrin alpha2. Tumour Biol. 2016;37(6):7951-7.

34. Zhou ZJ, Dai Z, Zhou SL, Hu ZQ, Chen Q, Zhao YM, Shi YH, Gao Q, Wu WZ Qiu SJ, Zhou J and Fan J. HNRNPAB Induces Epithelial-Mesenchymal Transition and Promotes Metastasis of Hepatocellular Carcinoma by Transcriptionally Activating SNAIL. Cancer Res. 2014;74(10):2750-62.

35. Sung JY, Park SY, Kim JH, Kang HG, Yoon JH, Na YS, Kim YN, Park BK Interferon consensus sequence-binding protein (ICSBP) promotes epithelialto-mesenchymal transition (EMT)-like phenomena, cell-motility, and invasion via TGF-beta signaling in U2OS cells. Cell Death Dis. 2014;5:e1224.

36. Liu C, Lin J. Long noncoding RNA ZEB1-AS1 acts as an oncogene in osteosarcoma by epigenetically activating ZEB1. Am J Transl Res. 2016;8(10): 4095-105.

37. Li M, Chen H, Zhao Y, Gao S, Cheng C. H19 Functions as a ceRNA in Promoting Metastasis Through Decreasing miR-200 s Activity in Osteosarcoma. DNA Cell Biol. 2016;35(5):235-40.

38. Lei $P$, Ding D, Xie J, Wang L, Liao Q, Hu Y. Expression profile of Twist, vascular endothelial growth factor and CD34 in patients with different phases of osteosarcoma. Oncol Lett. 2015;10(1):417-21.

39. Inoue $H$, Takahashi $H$, Hashimura M, Eshima K, Akiya M, Matsumoto T, Saegusa M. Cooperation of Sox4 with beta-catenin/p300 complex in transcriptional regulation of the Slug gene during divergent sarcomatous differentiation in uterine carcinosarcoma. BMC Cancer. 2016;16:53.

40. Pei $X, L v X, L i H$. Sox 5 induces epithelial to mesenchymal transition by transactivation of Twist1. Biochem Bioph Res Co. 2014;446(1):322-7.

41. Wang D, Han S, Wang X, Peng R, Li X. SOX5 promotes epithelialmesenchymal transition and cell invasion via regulation of Twist1 in hepatocellular carcinoma. Med Oncol. 2015;32(2):461.

\section{Submit your next manuscript to BioMed Central and we will help you at every step:}

- We accept pre-submission inquiries

- Our selector tool helps you to find the most relevant journal

- We provide round the clock customer support

- Convenient online submission

- Thorough peer review

- Inclusion in PubMed and all major indexing services

- Maximum visibility for your research

Submit your manuscript at www.biomedcentral.com/submit

) Biomed Central 